\title{
Agregados Inflables y Patrones Emergentes: Logro de Patrones de Orden Superior mediante Compresión Axial
}

\author{
Inflatable Aggregates and Emergent Patterns: Higher-Order Patterns Achievement through Axial \\ Compression
}

\author{
Pablo Salvador Banda \\ Non-Consensual Architectural Practice \\ Pontificia Universidad Católica de Chile \\ pablo.banda.p@hotmail.com
}

\author{
Arturo Lyon Gottlieb \\ Pontificia Universidad Católica de Chile \\ alyon@uc.cl
}

\begin{abstract}
This paper presents a Generative Design exploration of a simulated Inflatable Components Aggregate (ACN) ruled by local configurations and mechanical behavior, tested in a digital environment. The Design Product is revealed through compressions and deformations evaluation, where the ACN internal interaction's exhibits emerging global patterns.
\end{abstract}

Keywords: Generative design, Form Finding, Simulation.

\section{Introducción}

El desarrollo de diversas plataformas de modelación y simulación de formas y estructuras, ha gatillado la exploración de nuevas prácticas arquitectónicas, a través de procesos de diseño que buscan articular criterios físicos, programáticos, formales o contextuales. Las capacidades de software y sus aplicaciones, han sido determinantes en abrir nuevos alcances para procesos de diseño en arquitectura.

La simulación de propiedades física de comportamiento ha sido un extenso campo de exploración que ha definido nuevas relaciones entre arquitectos e ingenieros en los procesos de diseño. Métodos como el análisis de elementos finitos (FEA) han permitido evaluar formas complejas en su comportamiento físico, lo que ha permitido la materialización de emblemáticos edificios en las últimas décadas. También se ha explorado el uso FEA en el análisis de carga y estreses de Von Misses para la retro-alimentación sucesiva de formas embebiendo operaciones de diseño que incrementan la resistencia a la compresión al mismo tiempo que ampliando procesos de búsqueda de formal. (Al Haddad, 2007). La introducción de procesos optimización estructural evolutiva (ESO) en arquitectura, ha definido procesos en que el diseño de formas y su evaluación estructural están integradas y emergen de un mismo proceso generativo (Huang-Xie 2010 / Garcia-Lyon 2011).

Un lugar intermedio entre el uso de simulación con rigurosidad física y posibilidades creativas es planteado por un Diseño Basado en Físicas (Ramtin et al, 2012), en el cual la interacción digital con el material amplía las capacidades de un ejercicio análogo de form-finding logrando mayor complejidad en cuanto al incremento de componentes como también al incremento de restricciones materiales (Ramtin et al, 2012).

El caso presentado, basadi en elementos neumáticos, se refiere a una estructura de forma activa (Engel, 2007), en que las fuerzas físicas que actúan sobre el sistema definen directamente su forma; al variar las fuerzas varían consecuentemente las formas resultantes. El diseño de este tipo de estructura requiere de procesos de simulación de comportamiento físico y material para determinar configuraciones viables. Debido a lo anterior, en este caso es especialmente relevante la exploración de métodos de diseño generativo basados en comportamientos físicos del sistema. El trabajo acá detallado corresponde a una experiencia de racionalización material y form-finding multi-componente encarnada en un Agregado de Componentes Neumáticos (ACN) capaz de producir comportamientos globales emergentes derivados de la interacción entre sus partes.

La problemática fue levantada de un trabajo inicial (ver figura 1), en el cual se subdividió una mangade poliestireno de producción estandarizadas de $50 \mathrm{~cm}$ de ancho, incorporando diagonales termofusionadas para la obtención de componentes triangulares inflables e interconectados. Al agregar presión de aire, se produce una forma tridimensional estable y resistente definida por el efecto en cadena de la presión de un cojinete sobre sus cojines adyacentes determinan un comportamiento emergente en el sistema.

La anticipación digital para el logro de esta "forma estable" establece un problema complejo para la computación aplicada: la interacción en cadena entre componentes de forma variable incluso desafía la implementación del análisis finito debido a su naturaleza parcial, en ese sentido la rigurosidad de la simulación es tranzada por la obtención de un diseño emergente.

\section{Agregados y Patrones Emergentes}

Según Alexander (2005) existe una convergencia común en en las soluciones de diseño de los sistemas generativos: la incapacidad de formar órdenes mayores a los de su escala más próxima. En su texto, junto con plantear 15 propiedades armónicas de sistemas, establece que la relación con el contexto es clave para la conexión con el entorno y el encuentro de tales órdenes superiores (Alexander, 2005). 

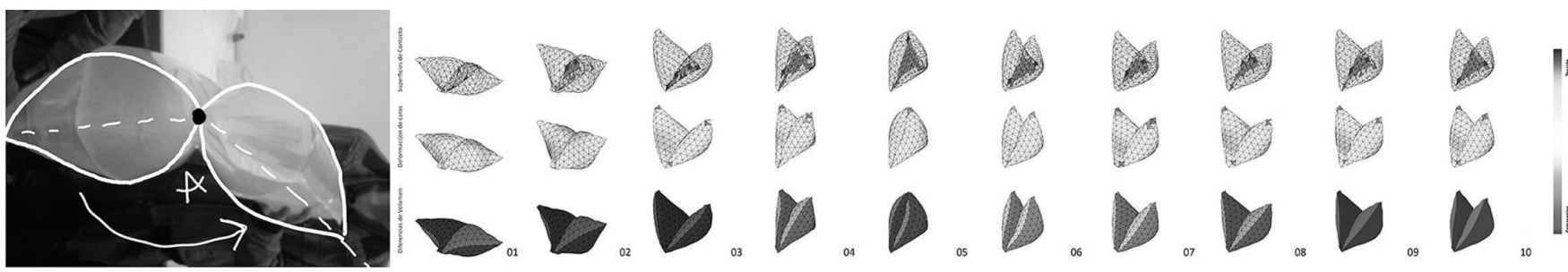

Figura 1: Interacción simple de cojines inflados, confección propia.

En este trabajo planteamos una pre-estructura para un agregado de componentes inflables, formando un contexto de pre-existente de relaciones, el cual mediante la carga manifiesta patrones globales interesantes que trascienden el estanco en el orden inmediato.

Aunque sea clara la manipulación y aislación del efecto a producir, indirectamente conlleva a un ambiente de examinación, en el cual se puede desarrollar una sensibilidad posiblemente exportable a otras experiencias menos restringidas.

\section{Objetivos}

La importancia de este ejercicio radica en el logro parcial o total de los siguientes objetivos:

1 Encontrar patrones de mayor orden en diseño generativo utilizando un entorno acotado y simple de elementos.

2 Ejecutar soluciones emergentes diseñando para el uso de materiales disponibles en el mercado y técnicas low-tech de fabricación.

3 Exacerbar el pliegue en las interacciones entre inflados.

Para el punto 02 los patrones globales luego de la carga del sistema son obtenidos solamente variando amarras dentro del sistema de mangas plásticas. El punto 03 corresponde a la visualización y valoración estética de los resultados

\section{Método}

La tarea de diseño suple la incapacidad de los actuales módulos de física para la simulación de un sistema de inflado de multicomponentes con la simulación en separado y por fases del problema. Esta aproximación ha sido introducida como válida, sin embargo según Dieter Ströbel (2012) el caso de simulación para tenso-estructuras los resultados serán mucho más ajustados si es que los comportamientos dinámicos son integrados en una sola simulación.

\section{Análisis previo: Interacción entre cojines}

La interacción entre dos cojines, el presionar uno con el otro, nos entrega un ángulo mínimo bajo una presión constante. Esto fue observado con cojines confeccionados con mangas plásticas de 50 $\mathrm{cm}$ termo-selladas, mientras en paralelo esto se simuló utilizando el motor de físicas Kangaroo dentro del plug-in de modelado paramétrico Grasshopper de Rhinoceros. Las pruebas de la figura 01 se realizaron girando los cojines con respecto a su borde común, haciéndolos chocar con un plano imaginario de modo de simular la zona de contacto.
La premisa de solucionar la interacción en masa de componentes inflados como un origami de ángulos restringidos fue descartada ya que se entendió la complejidad del traspaso de fuerzas gradientes rigidez en el sistema, a su vez, dicho motor de físicas no permitió la interacción directa de muchos componentes.

\section{Descripción de Sub-Tareas de Diseño}

En resumen, la tarea de diseño genera un patronaje en triángulos de una manga plástica, para luego inflarla, repetirla en forma de cilindro especificando nodos sellados entre mangas y luego ensaye. Esta rutina de trabajo se soluciona mediante 5 módulos, de los cuales 2 son de simulación propiamente tal, 2 de entradas geométricas y 1 corresponde a una interfaz de visualización (ver figura 3). En detalle cada módulo realiza las siguientes labores.

\section{Inflado de componentes}

Simula el inflado de componentes sin aumentar el área de la superficie, restricción devenida de la utilización de superficies plásticas pero sin elasticidad. Esta característica hace que nuestro ejercicio difiera de las actuales técnicas de form-finding en el cual alteras la concentración de áreas dentro de un paño para lograr superficies mínimas.

Poligonalización de teselas pre-inflado: Según Gerry D’Anza (2012), un paso primordial en un proceso de form-finding es establecer una poligonalización adecuada, debido a que la topología de los vértices en la malla de polígonos regirá las concentraciones de las áreas cuando la superficie se relaje. Aunque no se utilizó un proceso de relajación de superficies si no uno basado en resortes o springs, la confección de triángulos lo más parecidos posibles en una nube de puntos homogénea ayudó a mantener bajísimos incrementos de área al ser infladas las mangas.

Inflado de Cojines: Las mallas a inflar corresponden a cojines predispuestos en una manga triangulada. Para el inflado de las mangas de cojines se utilizó componente de físicas Kangaroo. Se procedió manteniendo en la medida de lo posible una distorsión de material menor al 3\% (teniendo en cuenta que el material se estira mínimamente), esto se logra mediante la inclusión de Springs en cada arista de la malla de polígonos con un Factor de Encogimiento de 0.9 como máximo con respecto a la medida original de las aristas forzando la mantención de área. 


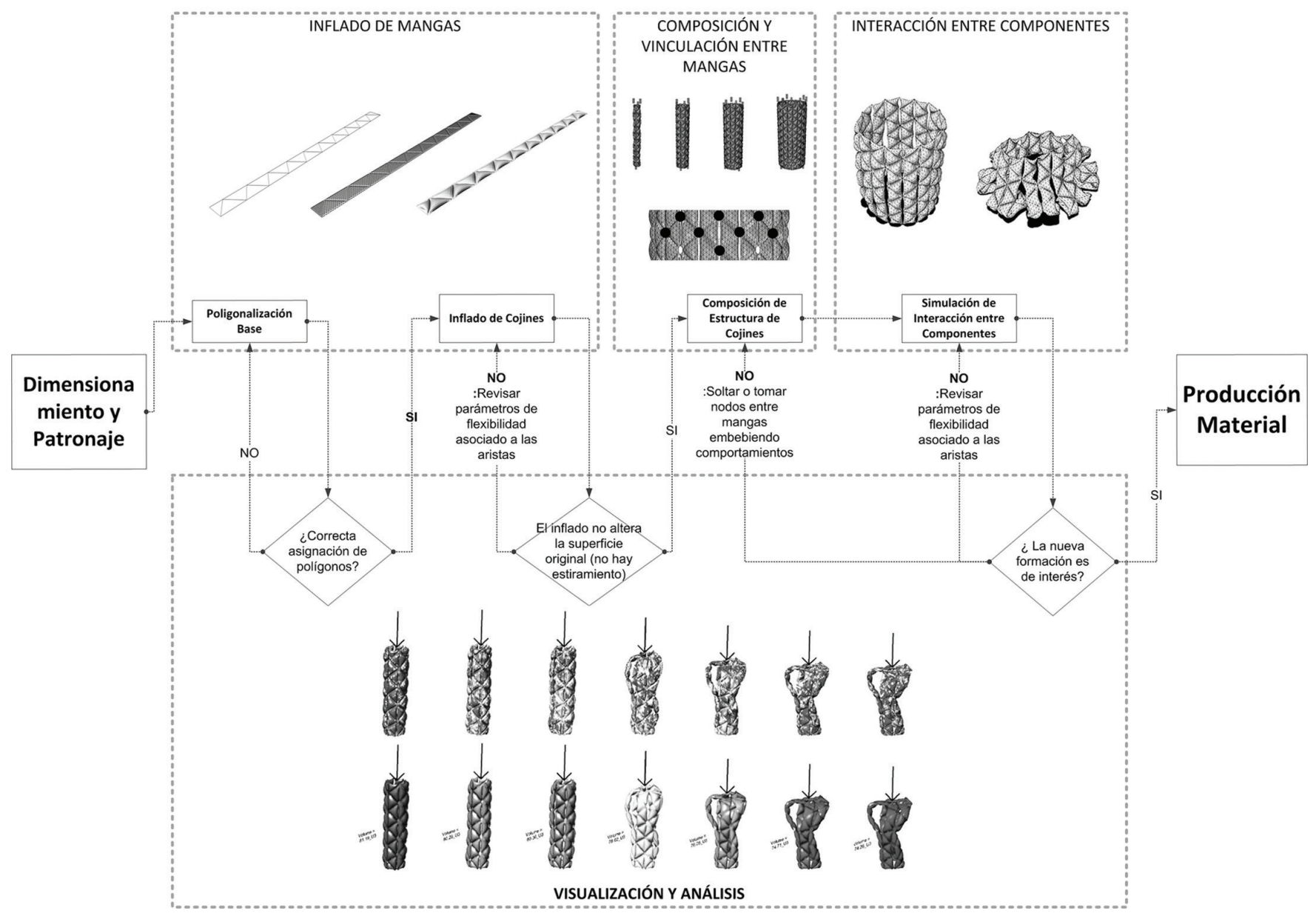

Figura 2: Tarea de Diseño del Agregado de Componentes Neumáticos, confección propia.

\section{Interacción entre componentes}

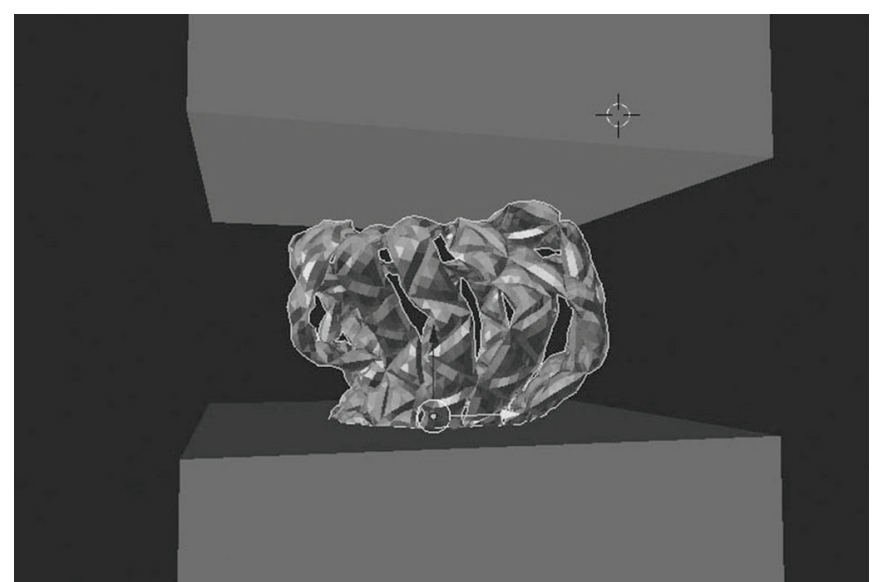

Figura 3: Carga de los cilindros en Blender, captura de pantalla

Debido a las limitaciones del componente de físicas Kangaroo en cuanto al manejo de interacción entre múltiples Soft Bodies, fue necesaria la implementación del motor de simulación dinámica del programa Blender. Éste motor de simulación se caracteriza por simplificar el trazo de springs.
El ensaye se realizó utilizando como referente el trabajo de ensaye de probetas de acero expandido de Smith, Graciano y Martinez (2009). En síntesis, el ensaye se produce con un cilíndro de mangas plásticas vinculadas, fijadas desde la base para ser cargadas por un volumen superior (ver figura 3). Este volumen en algunos casos se transforma en una densidad de carga: el sólido de carga intersecta y contienen la "probeta" cargándolo. Todas las mangas están unidas con nodos en los extremos.

\section{Resultados}

Bajo cierta tolerancia a eventos menores de intersección. En general se puede decir la importancia entre la relación del grosor de los cojines versus área de la sección controla la estabilidad del sistema: El aplaste ocurre generalmente en composiciones de 16 mangas plásticas, las composiciones con 8 mangas plásticas se resistieron produciendo un error de intersección con el sólido compresor. En específico para cada tipo se puede señalar lo siguiente:

Espirales Simples: La variación gradual de la altura donde los nodos se ubican hace que la composición acomode una especie de "animación desplegada" de la curvatura rotando a través del eje. 

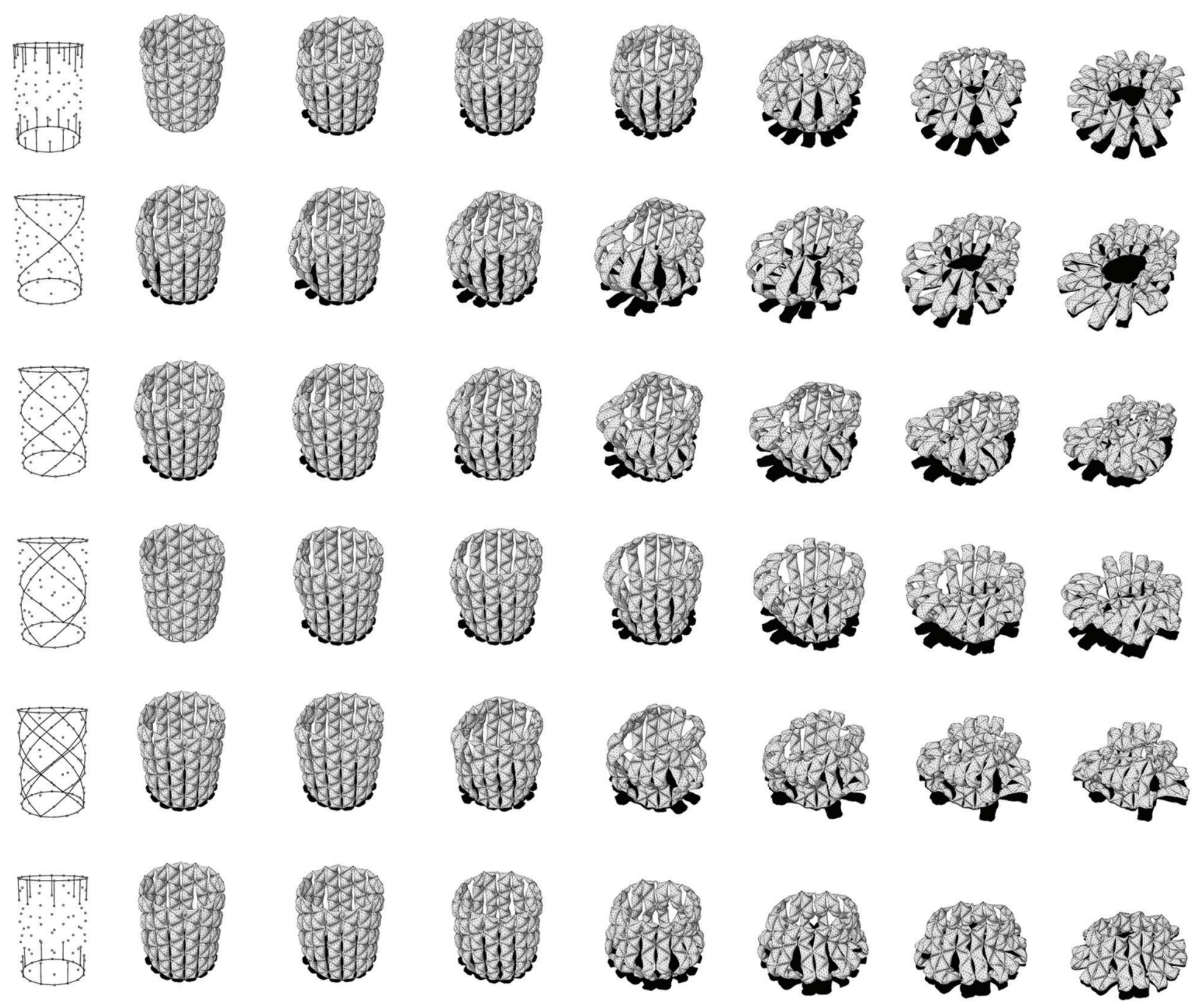

Figura 4: Tarea de Diseño del Agregado de Componentes Neumáticos, confección propia.

Las zonas de las mangas liberadas de nodos forman arcos, esto se radicaliza en el caso de la formación simple de un espiral en un cilíndro con 16 mangas donde algunas no tienen nodos entre los extremos. En casos donde siempre hay nodos entre los extremos se propician los arcos "hacia adentro".

Espirales Dobles: Dada la situación simétrica de los espirales Dobles y su forma tipo diagrid, estos islotes sin nodos con forma de diamantes propicia la aparición de contrastes cóncavoconvexos. La aparición de 2 nodos de control en cada manga establece 2 puntos de inflexión y 3 arqueamientos en cada manga, los cuales varían de cóncavo a convexo de forma intercalada. En casos en los cuales los nodos están más cercanos a la zona central es más ocurrente que se produzca una concavidad.

Agarre Intercalado entre extremos: Este tipo de distribución de nodos entregó formas más o menos estables, que destacan por separarse en pares de mangas, logrando en el caso de 16 mangas un "aplaste" recto a diferencia de la gran mayoría de los casos en que los cilindros tienden siempre hacia un lado.

\section{Trabajos Futuros}

Incorporación de variabilidad lateral: La aproximación del ejercicio posee ciertas limitaciones: la dirección vertical de los esfuerzos imperó en las diferencias de curvatura a través de la vertical. Excepción de esto son las rotaciones claras vistas en el caso de espirales simples y dobles, debido a que la relación con el cilindro en general es en ambos sentidos. Es parte de un trabajo futuro cambiar orientaciones de las mangas, re-definir la unidad manga misma y los sentidos de doblez en general.

Forma principal personalizable: Es deseable que la composición entre mangas y cojinetes pueda personalizarse antes o durante la simulación, es parte de un trabajo futuro también la incorporación de miembros rígidos y tensores en el sistema. 


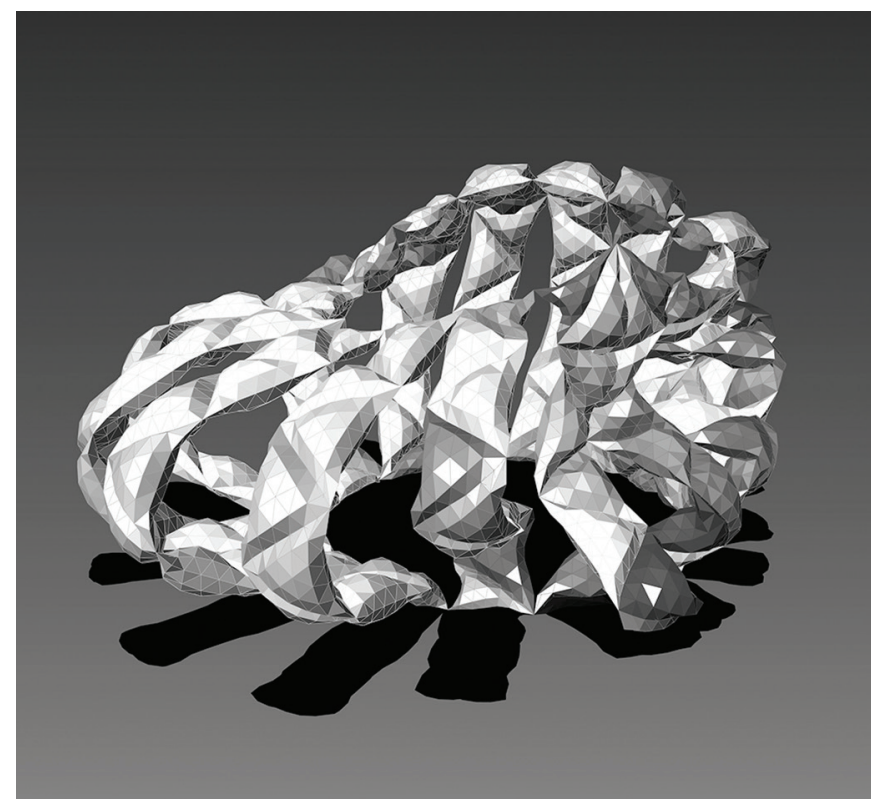

Figura 5: Cilindro con espiral simple, sombreado según compresión. Confección propia.

\section{Conclusión}

Un proceso de inflado y simulación de formaciones de componentes inflables no es tarea fácil, debido a la adecuación a técnicas presentes en los software, sobre todo si se está pasando desde un software a otro y estos poseen distintos propósitos. De acuerdo a los objetivos planteados se presentan las siguientes conclusiones:

\section{Dobleces y pliegues: Configuración de órdenes emergentes}

Se observaron 3 niveles de dobleces: entre cojinetes, en las mismas mangas y a través de la superficie del "cilindro" conformado por las mangas. Se observa una colaboración bidireccional de las partes y el todo:

1 Top-Down: La carga superior exige el acomodamiento y las curvas en el segundo nivel.

2 Middle-to-Extremes: Los patrones en la superficie del cilindro son delineados directamente por los nodos.

3 Bottom-Up: Desde los pliegues entre cojinetes hacia arriba se establece la orientación de las curvaturas (relaciones cóncavoconvexo.).

\section{Aproximación low-tech y racionalización}

La deformación de los elementos en cuanto a su patronaje original presenta rangos más que aceptables: para el inflado original se admitió una alteración máxima del área original del 3\%, en la carga de los cilindros se estima que la alteración del área varió desde ese 3 al $7 \%$.

\section{Proyección e implicancias}

Este trabajo ha mostrado ser capaz de producir patrones emergentes de alto rango para una topología fija, en su control en base a los nodos se encontraron tendencias geométricas $y$ comportamientos globales. (Alexander, 2005).

En si se ha desarrollado un método digital con una perspectiva estético-organicista, el que podría seguir siendo desarrollado y orientado hacia la interacción con el usuario a tiempo real. No obstante lo anterior debemos destacar el cambio de carácter de la simulación desde uno de comprobación y encuentro de información para retroalimentar los productos a uno que trabaja develando potencialidades expresivas (figura 05), vale decir estamos saliendo de un medio técnico a otro con profundas implicancias artísticas. Es tarea futura llevar este sistema al mundo real en una probeta a gran escala donde se varíe la topología y se infiera en vivo patrones sobre las formaciones de componentes inflados.

\section{Agradecimientos}

Agradecimientos al equipo del concurso interno de Tensoestructuras: María Quiroz, Alan Patrick, José Mujica y Diego Melo, del curso Tenso-estructuras Generativas dirigido por Arturo Lyon en la UC.

\section{Referencias}

Al Haddad, T. (2007). PerFORMance: "Integrating Structural Feedback into Design Processes for Complex Surface-Active Form, Tesis de Magister, Georgia Institute of Technology.

Alexander, C. (2005) Harmony-Seeking Computations. The Grand Challenge in Non-Classical Computation.

Attar, R.; Aish, R, Stam, S. et al., (2010) Embedded Rationality: A Unified Simulation Framework for Interactive Form Finding. International Journal of Architectural Computing, 8 (4), 399-418. D’Anza, G.; (2012), "ForTEN Software: Charla Técnica" Presentación Oral en el contexto del V Simposio Latinoamericano de Tensoestructuras, Santiago 2012.

Dieter, S.; (2012), "Easy Software: Charla Técnica" Presentación Oral en el contexto del V Simposio Latinoamericano de Tensoestructuras, Santiago 2012.

Engel, H; (2007), Structure Systems, Hatje Cantz,

Garcia, R. Lyon, A; (2011), "De la Optimizacion Estructural Evolutiva al Diseño Parametrico basado en Desempeño", SIGraDi 2012.

Smith, D. Graciano, C. Martinez, G.(2009) Estudio del Comportamiento de Estructuras Fabricadas con Metal Expandido Sometidas a Compresión Axial. Boletín Técnico IMME, 47(2), 01-26. 